\title{
Association of atrial fibrillation with outcomes in patients hospitalized with inflammatory bowel disease: an analysis of the National Inpatient Sample
}

\author{
Ebad Ur Rahman'1, Vijay Gayam², Pavani Garlapati², Neelkumar Patel², Fatima Farah³, \\ Adee El-Hamdani ${ }^{4}$, Arfaat Khan ${ }^{5}$, Paul I. Okhumale ${ }^{6}$, Wilbert S. Aronow ${ }^{7}$ Mehiar El-Hamdani ${ }^{6}$
}

\author{
'Department of Internal Medicine, St Mary's Medical Center, Huntington, WV, USA \\ 2Department of Internal Medicine, Interfaith Medical Center, Brooklyn, NY, USA \\ ${ }^{3}$ Department of Internal Medicine, Deccan College of Medical Sciences, Hyderabad, \\ India \\ ${ }^{4}$ Alleghany Health Network, Pittsburg, PA, USA \\ ${ }^{5}$ Department of Cardiology, Henry Ford Hospital, Detroi, MI, USA \\ ${ }^{6}$ Department of Cardiology, Marshall University, Huntington, WV, USA \\ 'Department of Cardiology, Westchester Medical Center and New York Medical \\ College, Valhalla, NY, USA
}

Submitted: 8 January 2021

Accepted: 26 January 2021

Arch Med Sci Atheroscler Dis 2021; 6: e40-e47

DOI: https://doi.org/10.5114/amsad.2021.105256

Copyright @ 2021 Termedia \& Banach

\section{Abstract}

Introduction: We aimed to determine in-hospital outcomes, length of hospital stay (LOS) and resource utilization in a contemporary cohort of patients with inflammatory bowel disease (IBD) and atrial fibrillation (AFIB).

Material and methods: The National Inpatient Sample database October 2015 to December 2017 was utilized for data analysis using the International Classification of Diseases, Tenth Revision codes to identify the patients with the principal diagnosis of IBD.

Results: Of 714,863 IBD patients, 64,599 had a diagnosis of both IBD and AFIB. We found that IBD patients with AFIB had a greater incidence of in-hospital mortality $(\mathrm{OR}=1.3 ; 95 \% \mathrm{Cl}: 1.1-1.4)$, sepsis $(\mathrm{OR}=1.2 ; 95 \% \mathrm{Cl}$ : $1.1-1.3)$, mechanical ventilation $(\mathrm{OR}=1.2 ; 95 \% \mathrm{Cl}: 1.1-1.5)$, shock requiring vasopressor $(\mathrm{OR}=1.4 ; 95 \% \mathrm{Cl}: 1.1-1.9)$, lower gastrointestinal bleeding (LGIB) $(\mathrm{OR}=1.09,95 \% \mathrm{Cl}: 1.04-1.1)$, and hemorrhage requiring blood transfusion (OR $=1.2,95 \% \mathrm{Cl}: 1.17-1.37)$. Mean LOS \pm SD, mean total charges and total costs were higher in patients with IBD and AFIB.

Conclusions: In this study, IBD with AFIB was associated with increased in-hospital mortality and morbidity, mean LOS and resource utilization.

Key words: inflammatory bowel disease, atrial fibrillation, NIS, propensitymatched, outcomes.

\section{Introduction}

Inflammatory bowel disease (IBD) is characterized by non-infectious chronic inflammation of the gastrointestinal tract, and primarily includes Crohn's disease (CD) (which can affect any segment of the gastrointestinal tract from the mouth to the anus), ulcerative colitis (UC) (which is limited to the colonic mucosa), and indeterminate colitis [1]. The incidence and prevalence of IBD are increasing worldwide with the peak of occurrence usually happening in the second to fourth decade of life, and a second smaller peak occurring between 50 and 70 years of age [1, 2]. IBD is

\author{
Corresponding author: \\ Dr. Ebad Ur Rahman \\ Department \\ of Internal Medicine \\ St Mary's Medical Center \\ Huntington, WV, USA \\ E-mail: drebadmd@gmail.com
}


associated with an increased risk of cardiovascular diseases such as stroke, myocardial infarction, and early atherosclerosis with increased intimal thickness of the common carotid artery [3, 4]. High levels of C-reactive protein (CRP), a major marker reflecting activity and severity of inflammation in IBD, are also associated with atherogenesis, atherosclerotic cardiovascular disease and atrial arrhythmia [5-7].

Atrial fibrillation (AFIB) is the most common type of arrhythmia observed in clinical practice, with an estimated prevalence of $0.4 \%$ to $1 \%$ in the general US population [8-10]. The burden of AFIB in the US has been on the rise, with 2.3 million adults with AFIB in 2000 to an expected value of 12.1 million adults in 2030 [10, 11]. AFIB mostly affects the elderly and is more prevalent in men and white Caucasians [10, 12, 13]. AFIB like other cardiac arrhythmias is significantly associated with increased risks of cardiovascular complications, consequently leading to decreased quality of life, disability, healthcare expenses, and high mortality [14]. Recently, inflammation is being recognized as a pathogenic contributor to the development of AFIB [15]. Previous research has shown a significant association between serum inflammatory mediators such as CRP, tumor necrosis factor- $\alpha$, interleukin (IL)-2, IL-6, and IL-8, and the development and persistence of AFIB [16, 17]. Several cardiovascular disorders, notably coronary atherosclerosis, are associated with inflammation, and cytokines are known to affect plaque rupture and thrombus formation, resulting in myocardial infarction [18].

Considering that pathogenesis of AFIB is being increasingly linked to systemic inflammation, IBD may be a potential risk factor for AFIB, and the co-existence of the diseases could substantially lead to worsening outcomes (such as disability, health care utilization, medical costs, mortality). However, there is limited information regarding the association between comorbid AFIB and in-hospital outcomes, length of hospital stays, and resource utilization in people with IBD. To date, there are no data on how AFIB affects inpatient outcomes of IBD patients who are at the greatest risk for frequent hospitalizations. In this paper, we aim to study these parameters and other outcomes from a population database.

\section{Material and methods}

\section{Study data}

In this retrospective analysis, we utilized the National Inpatient Sample (NIS) data from October 2015 to December 2017. The NIS database is sponsored by the Agency for Healthcare Research and
Quality as a part of the Healthcare Cost and Utilization Project (HCUP) and is the largest publicly available all-payer administrative database, containing data on more than 7 million hospitalizations (unweighted); when weighted, it represents about 35 million hospitalizations nationally. It provides information on clinical and resource utilization with safeguards to protect data for individual patients, physicians, and hospitals. Beginning in October 2015, the NIS started using the International Classification of Diseases, Tenth Edition, Clinical Modification/Procedure Coding System (ICD-10-CM/PCS) to reflect the implementation of ICD-10-CM/PCS by hospital systems. Using the Agency for Healthcare Research and Quality sampling and weighting method, national estimates of the entire US hospitalized population were calculated.

\section{Study design}

Given the de-identified nature of the NIS data, our study was exempt from approval from the Institutional Review Board. We identified all patients ( $\geq 18$ years of age) who had a principal diagnosis of IBD ( $n=714863)$, using their respective ICD-10CM/PCS codes. We divided the total sample into two groups: IBD with AFIB $(n=64599)$ and IBD alone $(n=650264)$. We identified patients with AFIB using appropriate diagnosis codes. The ICD10-CM/PCS codes used in this study are displayed in Supplementary Table SI.

For baseline characteristics, we used patient demographics (age, race, and sex), the Charlson comorbidity index, insurance status, hospital characteristics, and relevant co-morbidities coronary artery disease (CAD), hypertension (HTN), obesity, dyslipidemia, diabetes mellitus (DM), congestive heart failure, chronic lung disease, peripheral vascular disease, and chronic kidney disease (CKD) (Table I). Comorbidities were identified using their respective ICD-10-CM/PCS codes (Supplementary Table SI).

\section{Outcomes}

The primary outcome of interest was all-cause in-hospital mortality. Secondary outcomes included incidence of acute kidney injury (AKI), peritonitis, intestinal obstruction, lower gastrointestinal bleeding (LGIB), sepsis, acute coronary syndrome, shock requiring vasopressors, disseminated intravascular coagulation (DIC), hemorrhage requiring blood transfusion, venous thromboembolism (VTE), acute respiratory failure, colectomy, and mechanical ventilation. Complications were identified using their respective ICD-10-CM/PCS (Supplementary Table $\mathrm{SI}$ ). We also studied the length of hospital stay (LOS), hospital costs, and reimbursement. 
Ebad Ur Rahman, Vijay Gayam, Pavani Garlapati, Neelkumar Patel, Fatima Farah, Adee El-Hamdani, Arfaat Khan, Paul I. Okhumale, Wilbert S. Aronow, Mehiar El-Hamdani

Table I. Demographics comparing IBD with AFIB vs. IBD alone

\begin{tabular}{|c|c|c|c|}
\hline Variable & IBD with AFIB (\%) & IBD without AFIB (\%) & $P$-value \\
\hline Total & 64599 & 650264 & \\
\hline Age (mean \pm SD) & $73.2 \pm 11.8$ & $50.8 \pm 19.5$ & $<0.001^{*}$ \\
\hline Female & 52.3 & 55.2 & 0.22 \\
\hline \multicolumn{4}{|l|}{ Race (\%): } \\
\hline Caucasian & 88.8 & 77.2 & \\
\hline African American & 5.1 & 12.1 & \\
\hline Hispanic & 3.0 & 6.5 & $<0.001^{*}$ \\
\hline Asian & 0.9 & 1.2 & \\
\hline Native American & 0.2 & 0.4 & \\
\hline Others & 1.7 & 2.4 & \\
\hline \multicolumn{4}{|l|}{ Hospital bed size (\%): } \\
\hline Small & 17.7 & 18.4 & \\
\hline Medium & 30.1 & 27.8 & 0.11 \\
\hline Large & 52.1 & 53.7 & \\
\hline \multicolumn{4}{|l|}{ Hospital region (\%): } \\
\hline Northeast & 23.6 & 21.6 & \\
\hline Midwest & 23.7 & 24.5 & 0.06 \\
\hline South & 34.1 & 36.6 & \\
\hline West & 18.4 & 17.1 & \\
\hline \multicolumn{4}{|l|}{ Discharge: } \\
\hline Routine & 72.1 & 42.7 & $<0.001^{*}$ \\
\hline Skilled nursing facility & 1.9 & 2.8 & \\
\hline \multicolumn{4}{|c|}{ Charlson comorbidity index (\%): } \\
\hline 0 or 1 & 31.8 & 70.9 & \\
\hline 2 & 19.3 & 11.7 & $<0.001^{*}$ \\
\hline 3 & 48.8 & 17.2 & \\
\hline \multicolumn{4}{|l|}{ Insurance type (\%): } \\
\hline Medicare & 36.5 & 36.5 & \\
\hline Medicaid & 3.6 & 16.9 & $<0.001^{*}$ \\
\hline Private & 13.97 & 39.8 & \\
\hline Uninsured & 0.8 & 3.67 & \\
\hline Teaching hospital & 69.9 & 70.9 & 0.32 \\
\hline \multicolumn{4}{|l|}{ Chronic co-morbidity (\%): } \\
\hline $\mathrm{DM}$ & 30.5 & 15.0 & $<0.001^{*}$ \\
\hline HTN & 40.3 & 30.3 & $<0.001^{*}$ \\
\hline CAD & 36.47 & 9.8 & $<0.001^{*}$ \\
\hline Anemia & 7.7 & 8.1 & 0.13 \\
\hline Obesity & 12.2 & 11.2 & 0.15 \\
\hline Dyslipidemia & 45.2 & 40.9 & 0.53 \\
\hline CKD & 25.7 & 8.7 & $<0.001^{*}$ \\
\hline $\mathrm{CHF}$ & 28.5 & 4.9 & $<0.001^{*}$ \\
\hline CLD & 14.6 & 13.5 & 0.11 \\
\hline PVD & 6.2 & 4.1 & 0.23 \\
\hline
\end{tabular}

"Statistically significantly different result. DM - diabetes mellitus, HTN - hypertension, CAD - coronary artery disease, CHF - congestive heart failure, PVD - peripheral vascular disease, CLD - chronic lung disease. 


\section{Statistical analysis}

We conducted all statistical analyses as per the recommended methods accounting for the intricate survey design of the NIS database using STATA 15.0 (Stata Corp LLC). Categorical data are reported as frequency and percentage, and continuous data as mean with standard deviation and standard error. Pearson's $\chi^{2}$ test was used to determine significant differences in categorical variables, and continuous variables were analyzed using Student's t-test. Univariable logistic regression analysis was used to calculate unadjusted odds ratios (OR) for the primary and secondary outcomes; then multivariable logistic regression analysis was performed for potential confounders. The multivariate logistic regression model was built by using only variables that were associated with the outcome of interest in univariable regression analysis at $p<0.2$. All analyses in our study were weighted using provided discharge weights to produce national estimates. Statistical significance was set at a two-sided $p$-value of $<0.05$. Reported hospital costs and charges were inflation-adjusted for July 2020 using the Consumer Price Index (provided by the U.S. Department of Labor). Sensitivity analysis was performed, excluding the population with CHF and CAD, to determine the accuracy of the results. A full list of covariates used in the regression analysis and confounders in the multivariable regression model is shown in Supplementary Table SI.

\section{Results}

\section{Characteristics of the study population}

We identified 714,863 patients admitted with the diagnosis of IBD. Of these, 64,599 had concomitant AFIB, and 626,869 had IBD only. The mean age (SD) in the IBD + AFIB group and IBD group was 73.2 (11.8) years and $50.8 \pm 19.5$ years, respectively. There was a significant difference between groups for age, race, discharge disposition, Charlson comorbidity index, insurance, DM, HTN, CAD, CKD, and CHF (Table I). There was a higher percentage of patients with DM, HTN, CKD, CAD, and $\mathrm{CHF}$ in patients with IBD + AFIB.

\section{Comparison of primary and secondary outcomes}

The proportion of primary and secondary outcomes in both groups is shown in Table II. We compared the outcomes between IBD with AFIB and IBD groups. In the univariate analysis, a statistically significant difference was observed for in-hospital mortality $(\mathrm{OR}=4.1,95 \% \mathrm{Cl}$ : 3.7-4.5), AKI $(O R=2.6,95 \% \mathrm{Cl}: 2.5-2.7)$, sepsis $(\mathrm{OR}=$ 2.3, $95 \% \mathrm{Cl}: 2.1-2.4)$, peritonitis $(\mathrm{OR}=1.2,95 \%$
$\mathrm{Cl}: 1.05-1.5)$, intestinal obstruction $(\mathrm{OR}=0.85$, $95 \% \mathrm{Cl}: 0.57-0.75)$, colectomy $(\mathrm{OR}=0.5,95 \% \mathrm{Cl}$ : 0.4-0.6), DIC (OR $=2.1,95 \% \mathrm{Cl}: 1.5-2.9)$, acute respiratory failure $(\mathrm{OR}=2.5,95 \% \mathrm{Cl}: 2.3-2.8)$, mechanical ventilation $(\mathrm{OR}=2.8,95 \% \mathrm{Cl}: 2.5-3.1)$, shock requiring vasopressor $(\mathrm{OR}=3.4,95 \% \mathrm{Cl}$ : 2.8-4.2), LGIB (OR = 1.4, 95\% Cl: 1.3-1.5), hemorrhage requiring blood transfusion $(\mathrm{OR}=3.4$, $95 \% \mathrm{Cl}: 3.0-3.8)$, acute myocardial infarction $(\mathrm{OR}=3.4,95 \% \mathrm{Cl}: 3.0-3.8)$ and venous thromboembolism (OR $=1.6,95 \% \mathrm{Cl}: 1.3-1.9)$. Except intestinal obstruction and colectomy, which were lower, all other complications were higher in the IBD with AFIB group.

Multivariable logistic regression was performed to adjust for potential confounders. After adjusting for confounders, IBD patients with AFIB had a significantly greater incidence of in-hospital mortality $(\mathrm{OR}=1.3 ; 95 \% \mathrm{Cl}: 1.1-1.4)$, sepsis $(\mathrm{OR}=1.2 ; 95 \% \mathrm{Cl}: 1.1-1.3)$, mechanical ventilation $(\mathrm{OR}=1.2 ; 95 \% \mathrm{Cl}: 1.1-1.5)$, shock requiring vasopressor $(\mathrm{OR}=1.4 ; 95 \% \mathrm{Cl}$ : 1.1-1.9), LGIB $(\mathrm{OR}=1.09,95 \% \mathrm{Cl}: 1.04-1.1)$, and hemorrhage requiring blood transfusion $(\mathrm{OR}=1.2,95 \% \mathrm{Cl}$ : 1.17-1.37) (Table II).

A sensitivity analysis where patients with CAD and CHF were excluded to assess whether AFIB was still associated with increased risk of mortality and complications in univariable and multivariable analyses was performed. The results were similar, with increased in-hospital mortality $(\mathrm{OR}=$ 1.3; $95 \% \mathrm{Cl}: 1.1-1.5)$, sepsis $(\mathrm{OR}=1.2 ; 95 \% \mathrm{Cl}$ : $1.1-1.4)$, mechanical ventilation $(\mathrm{OR}=1.2 ; 95 \% \mathrm{Cl}$ : $1.1-1.4)$, shock requiring vasopressor $(\mathrm{OR}=1.5$; 95\% Cl: 1.1-1.9), LGIB (OR = 1.08, 95\% Cl: 1.03$1.1)$, and hemorrhage requiring blood transfusion $(\mathrm{OR}=1.2,95 \% \mathrm{Cl}: 1.1-1.3)$ (Table III).

\section{Factors associated with mortality in IBD + AFIB group}

Multivariate logistic regression was performed to identify the factors associated with mortality in the IBD + AFIB group. Advanced age (OR = 1.05; $95 \% \mathrm{Cl}: 1.04-1.06)$, congestive heart failure $(\mathrm{OR}=$ 1.7; $95 \% \mathrm{Cl}: 1.3-2.2)$, and CAD (OR $=1.5 ; 95 \% \mathrm{Cl}$ : 1.1-1.9) were identified as factors associated with mortality in IBD with AFIB (Table IV).

\section{Length of stay and resource utilization}

Statistically significance differences were observed in the mean length of stay (LOS), mean cost of care, and mean total charges between the two groups. Mean LOS ( \pm SD) $(6.7 \pm 7$ vs. $5.2 \pm 6.7, p<$ $0.001)$, mean total charges (\$ 76104 vs. $\$ 54876$, $p<0.001$ ), and mean total costs ( $\$ 18926$ vs. $\$ 13881$ ) were higher in IBD with AFIB (Table V). 
Ebad Ur Rahman, Vijay Gayam, Pavani Garlapati, Neelkumar Patel, Fatima Farah, Adee El-Hamdani, Arfaat Khan, Paul I. Okhumale, Wilbert S. Aronow, Mehiar El-Hamdani

Table II. Comparison of primary and secondary outcomes: IBD with AFIB vs. IBD

\begin{tabular}{|c|c|c|c|c|c|c|}
\hline \multirow[t]{2}{*}{ Variable } & \multicolumn{2}{|c|}{ Incidence (\%) } & \multirow{2}{*}{$\begin{array}{l}\text { Unadjusted } \\
\text { OR }(95 \% \mathrm{Cl})\end{array}$} & \multirow[t]{2}{*}{$P$-value } & \multirow{2}{*}{$\begin{array}{c}\text { Adjusted } \\
\text { OR }(95 \% \mathrm{Cl})\end{array}$} & \multirow[t]{2}{*}{$P$-value } \\
\hline & $\begin{array}{l}\text { IBD with } \\
\text { AFIB }\end{array}$ & $\begin{array}{l}\text { IBD with- } \\
\text { out AFIB }\end{array}$ & & & & \\
\hline In-hospital mortality & 4.7 & 1.1 & $4.1(3.7-4.5)$ & $<0.001^{*}$ & $1.3(1.1-1.4)$ & $<0.001^{\star}$ \\
\hline AKI & 25.0 & 11.2 & $2.6(2.5-2.7)$ & $<0.001^{*}$ & $1.0(0.97-1.09)$ & 0.31 \\
\hline Sepsis & 10.2 & 4.7 & $2.3(2.1-2.4)$ & $<0.001^{*}$ & $1.2(1.1-1.3)$ & $<0.001^{\star}$ \\
\hline Peritonitis & 0.8 & 0.6 & $1.2(1.05-1.5)$ & $0.01^{\star}$ & $1.05(0.83-1.3)$ & 0.63 \\
\hline Intestinal obstruction & 1.7 & 2.1 & $0.85(0.57-0.75)$ & $<0.001^{\star}$ & $0.94(0.82-1.07)$ & 0.39 \\
\hline Colectomy & 2.2 & 4.1 & $0.5(0.4-0.6)$ & $<0.001^{*}$ & $0.91(0.79-1.04)$ & 0.20 \\
\hline DIC & 0.3 & 0.1 & $2.1(1.5-2.9)$ & $<0.001^{*}$ & $1.1(0.75-1.6)$ & 0.6 \\
\hline Acute respiratory failure & 3.2 & 1.2 & $2.5(2.3-2.8)$ & $<0.001^{*}$ & $1.1(0.96-1.3)$ & 0.12 \\
\hline Mechanical ventilation & 4.2 & 1.5 & $2.8(2.5-3.1)$ & $<0.001^{\star}$ & $1.2(1.1-1.5)$ & $0.001^{*}$ \\
\hline Pressor requirements & 1.1 & 0.3 & $3.4(2.8-4.2)$ & $<0.001^{*}$ & $1.4(1.1-1.9)$ & $0.001^{*}$ \\
\hline LGIB & 39.1 & 30.81 & $1.4(1.3-1.5)$ & $<0.001^{*}$ & $1.09(1.04-1.1)$ & $<0.001^{\star}$ \\
\hline Blood requirement & 10.0 & 5.8 & $1.8(1.6-1.9)$ & $<0.001^{*}$ & $1.2(1.17-1.37)$ & $<0.001^{*}$ \\
\hline AMI & 3.6 & 1.0 & $3.4(3.0-3.8)$ & $<0.001^{*}$ & $0.92(0.80-1.05)$ & 0.24 \\
\hline VTE & 1.0 & 0.6 & $1.6(1.3-1.9)$ & $<0.001^{\star}$ & $1.01(0.81-1.2)$ & 0.92 \\
\hline
\end{tabular}

*Statistically significantly different results. AKI - acute kidney injury, DIC - disseminated intravascular coagulation, LGIB - lower gastrointestinal bleeding, AMI - acute myocardial infarction, VTE - venous thromboembolism. Variables used in the multivariate logistic regression: age, sex, race, Charlson comorbidity index, congestive heart failure, acute kidney injury, chronic kidney disease, anemia, shock requiring vasopressor, coronary artery disease, chronic lung disease, hypertension, dyslipidemia diabetes mellitus, disseminated intravascular coagulation, acute respiratory failure, peritonitis, colectomy, mechanical ventilation, venous thromboembolism, acute myocardial infarction, hemorrhage requiring blood transfusion, sepsis and intestinal obstruction.

Table III. Sensitivity analysis comparing primary and secondary outcomes: IBD with AFIB vs. IBD

\begin{tabular}{|lcccccc|}
\hline Variable & \multicolumn{2}{c}{ Incidence (\%) } & Unadjusted & $P$-value & $\begin{array}{c}\text { Adjusted } \\
\text { OR (95\% CI) }\end{array}$ & $P$-value \\
\cline { 2 - 6 } & $\begin{array}{c}\text { IBD with } \\
\text { AFIB }\end{array}$ & $\begin{array}{c}\text { IBD with- } \\
\text { out AFIB }\end{array}$ & & & & \\
\hline In-hospital mortality & 4.6 & 1.1 & $4.2(3.8-4.7)$ & $<0.001^{*}$ & $1.3(1.1-1.5)$ & $0.001^{*}$ \\
\hline AKI & 24 & 10.9 & $2.6(2.5-2.7)$ & $<0.001^{*}$ & $1.03(0.97-1.1)$ & 0.23 \\
\hline Sepsis & 10.3 & 4.6 & $2.3(2.1-2.5)$ & $<0.001^{*}$ & $1.2(1.1-1.4)$ & $<0.001^{*}$ \\
\hline Peritonitis & 0.8 & 0.6 & $1.2(1.05-1.6)$ & $0.01^{*}$ & $1.02(0.80-1.3)$ & 0.81 \\
\hline Intestinal obstruction & 1.9 & 2.3 & $0.91(0.58-0.78)$ & $<0.001^{*}$ & $0.96(0.92-1.03)$ & 0.07 \\
\hline Colectomy & 2.4 & 3.9 & $0.5(0.5-0.64)$ & $<0.001^{*}$ & $0.89(0.77-1.03)$ & 0.14 \\
\hline DIC & 0.4 & 0.2 & $2.3(1.6-3.2)$ & $<0.001^{*}$ & $1.1(0.79-1.7)$ & 0.43 \\
\hline Acute respiratory failure & 3.2 & 1.2 & $2.6(2.3-3.0)$ & $<0.001^{*}$ & $1.1(0.97-1.3)$ & 0.09 \\
\hline Mechanical ventilation & 4.2 & 1.5 & $2.8(2.5-3.2)$ & $<0.001^{*}$ & $1.2(1.1-1.4)$ & $0.01^{*}$ \\
\hline Pressor requirements & 1.1 & 0.3 & $3.3(2.7-4.2)$ & $<0.001^{*}$ & $1.5(1.1-1.9)$ & $0.003^{*}$ \\
\hline LGIB & 39.1 & 30.7 & $1.4(1.3-1.5)$ & $<0.001^{*}$ & $1.08(1.03-1.1)$ & $<0.001^{*}$ \\
\hline Blood requirement & 9.7 & 5.7 & $1.7(1.6-1.9)$ & $<0.001^{*}$ & $1.2(1.1-1.3)$ & $<0.001^{*}$ \\
\hline AMI & 3.1 & 0.9 & $3.3(3.0-3.8)$ & $<0.001^{*}$ & $1.01(0.86-1.17)$ & 0.88 \\
\hline VTE & 1.1 & 0.7 & $1.6(1.3-2.0)$ & $<0.001^{*}$ & $1.01(0.77-1.2)$ & 0.88 \\
\hline
\end{tabular}

*Statistically significantly different result. AKI - acute kidney injury, DIC - disseminated intravascular coagulation, LGIB - lower gastrointestinal bleeding. AMI - acute myocardial infarction, VTE - venous thromboembolism. Variables used in the multivariate logistic regression: age, sex, race, Charlson comorbidity index, acute kidney injury, chronic kidney disease, anemia, shock requiring vasopressor, hypertension, dyslipidemia diabetes mellitus, chronic lung disease, disseminated intravascular coagulation, acute respiratory failure, peritonitis, colectomy, mechanical ventilation, venous thromboembolism, acute myocardial infarction, hemorrhage requiring blood transfusion, sepsis and intestinal obstruction. 
Table IV. Factors associated with mortality in IBD with AFIB

\begin{tabular}{|lccc|}
\hline Variable & Odds ratio & $P$-value & $95 \% \mathrm{Cl}$ \\
\hline Advanced age & 1.05 & $<0.001^{*}$ & $1.04-1.06$ \\
\hline Congestive heart failure & 1.7 & $<0.001^{*}$ & $1.3-2.2$ \\
\hline CAD & 1.5 & $0.004^{*}$ & $1.1-1.9$ \\
\hline
\end{tabular}

*Statistically significantly different result. Variables used in the multivariate logistic regression: age, sex, race, acute myocardial infarction, insurance status, hospital bed size, hospital location, discharge disposition, teaching status of hospital, Charlson comorbidity index, hypertension, diabetes mellitus, peripheral vascular disease, chronic kidney disease, coronary artery disease, obesity dyslipidemia, congestive heart failure, chronic lung disease, acute kidney injury, peritonitis, disseminated intravascular coagulation, acute respiratory failure, peritonitis, colectomy, mechanical ventilation, venous thromboembolism, hemorrhage requiring blood transfusion, sepsis and intestinal obstruction.

Table V. Analysis for length of stay and cost of care

\begin{tabular}{|lccc|}
\hline Parameter & IBD with AFIB & IBD without AFIB & $P$-value \\
\hline Mean \pm SD LOS [days] & $6.7 \pm 7.0$ & $5.2 \pm 6.7$ & $<0.001^{\star}$ \\
\hline Mean total charge [\$] & 76104 & 54876 & $<0.001^{\star}$ \\
\hline Mean total cost [\$] & 18926 & 13881 & $<0.001^{\star}$ \\
\hline
\end{tabular}

*Statistically significantly different result.

The factors associated with increased length of stay in IBD with AFIB were advanced age (adjusted mean difference $=1.2,95 \% \mathrm{Cl}: 1.2-3.6)$, AKI (adjusted mean difference $=2.9,95 \% \mathrm{Cl}: 1.6-2.4$ ), sepsis (adjusted mean difference $=7.7,95 \% \mathrm{Cl}$ : 3.6-4.9), and VTE (adjusted mean difference $=1.6$, 95\% Cl: 2.8-6.1) (Table V).

\section{Discussion}

The main findings of our current investigation are as follows: (1) IBD patients with comorbid AFIB have increased mortality compared to IBD patients without AFIB, and this difference persisted after adjusting for potential confounders. (2) The presence of AFIB is an independent predictor of mortality in IBD patients even after excluding those with CHF and CAD, and about $9 \%$ of patients in our cohort have AFIB. (3) Advanced age, $C A D$, and CHF were also independently associated with death among IBD patients with AFIB. (4) IBD and AFIB patients have increased risk of sepsis and LGIB, higher cost of hospitalization and increased length of stay compared to IBD patients without AFIB even after adjusting for known confounders.

AFIB is a complex disease with several potential mechanisms. The prevalence of AFIB in our cohort of IBD patients is $9 \%$, which approximates $11.3 \%$ reported by Pattanshetty et al. [19] in 141 IBD patients, and significantly higher than the general US population of $0.95 \%$ as reported by Go et al. [10]. Several studies have shown that the inflammatory process is one of the mechanisms for the occurrence of AFIB [15, 20, 21]. Frustaci et al. demonstrated inflammatory changes in atrial tissues obtained from patients with isolated persistent AFIB [20]. Gedikli et al. found a 2 to 3 -fold increase in the presence of serum inflammatory markers in AFIB patients compared with controls [22]. It is suggested that inflammation contributes to both occurrence and persistence of AFIB [23]. Inflammation is thought to cause tissue damage by ischemia and oxidative stress, progressively leading to loss of atrial muscle mass with interstitial fibrosis and resulting in structural remodeling [22]. This process also impairs intracellular calcium current, resulting in atrial electrical remodeling, which are known determinants of AFIB [22].

IBD is characterized by chronic inflammation of the digestive tract, affecting the most productive age groups of the population [1, 2]. Proinflammatory cytokines have been involved in regulating the intestinal immune response, causing tissue injury, and mediating complications of IBD [18, 24]. Inflammatory markers such as IL6 have been reproducibly detected in serum of IBD patients and correlate with disease activity [24-28]. IL-6 stimulates the proliferation of mature T cells, enhances the differentiation of cytotoxic $T$ lymphocytes and affects the terminal differentiation and immunoglobulin production of $B$ cells and induces acute phase proteins [29, 30].

IBD patients are more prone to developing $\mathrm{AFIB}$ and the co-occurrence of the two diseases could lead to worse outcomes and frequent hospitalizations. This is the first study demonstrating additive effects of AFIB in the population of IBD patients. There is a reasonable body of evidence to support the pathophysiological features for the co-occurrence of IBD and AFIB [19, 29]. The higher 
prevalence of AFIB in the IBD population as reported in our study could be attributed to systemic inflammation. Systemic inflammation is known to be a significant contributor to the development of AFIB $[15,30]$. Several previous studies have suggested that systemic inflammation is linked to various pathological processes such as oxidative stress, apoptosis, and fibrosis of cardiomyocytes, all of which lead to structural and electrical remodeling of the atria, promoting the development and persistence of AFIB [30-32]. Moreover, increases in the level of serum inflammatory markers, such as CRP and IL-6, were observed in patients with AFIB and IBD, especially CD $[16,17]$.

In our national cohort of IBD patients, we have also demonstrated that AFIB is associated with worse in-patient survival and that difference persists despite accounting for confounding variables. We also demonstrated that AFIB is an independent predictor of mortality in IBD patients. The strong association of worse outcomes of IBD patients with AFIB poses unique management challenges.

Additionally, the association of worse mortality in IBD patients with AFIB also calls into question measures to screen for AFIB in this patient population. Timely detection of AFIB and subsequent implementation of relevant therapeutic measures could result in improved outcomes in IBD patients.

This study is observational, and residual measured and unmeasured confounding factors may influence these findings. The NIS is an administrative claim-based database that use ICD-10 CM codes for disease diagnosis that may be subject to error. Secondly, the NIS collects data on in-patient discharges, and each admission is registered as an independent event. It is possible that the same patient may have more than one subsequent admission over time. NIS samples are not designed to follow patients longitudinally, so long-term outcomes could not be assessed from the present dataset. Additionally, data on AFIB management are lacking from the NIS, which has important implications for the conclusions drawn from the study.

In conclusion, our study shows AFIB to be associated with worse outcomes in IBD patients. Given the higher prevalence of AFIB in IBD and a significantly higher incidence of mortality and morbidity in IBD patients with AFIB, it is imperative that treating physicians should have clinical suspicion for AFIB in this specific patient cohort, as timely AFIB detection could result in improved outcomes. Further longitudinal studies are required to explore the temporal trends of AFIB and its comorbidities in IBD patients, and to establish associations with mortality and morbidity due to the arrhythmia complications.

\section{Conflict of interest}

The authors declare no conflict of interest.

\section{References}

1. Thia KT, Loftus EV Jr, Sandborn WJ, Yang SK. An update on the epidemiology of inflammatory bowel disease in Asia. Am J Gastroenterol 2008; 103: 3167-82.

2. Papadakis KA, Targan SR. Role of cytokines in the pathogenesis of inflammatory bowel disease. Annu Rev Med 2000; 51: 289-98.

3. Papa A, Danese S, Urgesi R, et al. Early atherosclerosis in patients with inflammatory bowel disease. Eur Rev Med Pharmacol Sci 2006; 10: 7-11.

4. Rungoe C, Basit S, Ranthe MF, Wohlfahrt J, Langholz E, Jess T. Risk of ischaemic heart disease in patients with inflammatory bowel disease: a nationwide Danish cohort study. Gut 2013; 62: 689-94.

5. Pearson TA, Mensah GA, Alexander RW, et al. Markers of inflammation and cardiovascular disease: application to clinical and public health practice: A statement for healthcare professionals from the Centers for Disease Control and Prevention and the American Heart Association. Circulation 2003; 107: 499-511.

6. Poullis AP, Zar S, Sundaram KK, et al. A new, highly sensitive assay for C-reactive protein can aid the differentiation of inflammatory bowel disorders from constipation- and diarrhoea-predominant functional bowel disorders. Eur J Gastroenterol Hepatol 2002; 14: 409-12.

7. Chung MK, Martin DO, Sprecher D, et al. C-reactive protein elevation in patients with atrial arrhythmias: inflammatory mechanisms and persistence of atrial fibrillation. Circulation 2001; 104: 2886-91.

8. National Heart L, And Blood Institute Arrhythmia 2020.

9. Chugh SS, Blackshear JL, Shen WK, Hammill SC, Gersh BJ. Epidemiology and natural history of atrial fibrillation: clinical implications. J Am Coll Cardiol 2001; 37: 371-8.

10. Go AS, Hylek EM, Phillips KA, et al. Prevalence of diagnosed atrial fibrillation in adults: national implications for rhythm management and stroke prevention: the AnTicoagulation and Risk Factors in Atrial Fibrillation (ATRIA) Study. JAMA 2001; 285: 2370-5.

11. Prevention CFDCA. Atrial Fibrillation 2020.

12. Blackshear JL, Kopecky SL, Litin SC, Safford RE, Hammill SC. Management of atrial fibrillation in adults: prevention of thromboembolism and symptomatic treatment. Mayo Clin Proc 1996; 71: 150-60.

13. Rosamond W, Flegal K, Furie K, et al. Heart disease and stroke statistics--2008 update: a report from the American Heart Association Statistics Committee and Stroke Statistics Subcommittee. Circulation 2008; 117: e25146.

14. Murakoshi N, Aonuma K. Epidemiology of arrhythmias and sudden cardiac death in Asia. Circ J 2013; 77: 2419-31.

15. Aviles RJ, Martin DO, Apperson-Hansen C, et al. Inflammation as a risk factor for atrial fibrillation. Circulation 2003; 108: 3006-10.

16. Patel P, Dokainish H, Tsai P, Lakkis N. Update on the association of inflammation and atrial fibrillation. J Cardiovasc Electrophysiol 2010; 21: 1064-70.

17. Psychari SN, Apostolou TS, Sinos L, Hamodraka E, Liakos G, Kremastinos DT. Relation of elevated C-reactive protein and interleukin-6 levels to left atrial size and duration of episodes in patients with atrial fibrillation. Am J Cardiol 2005; 95: 764-7. 
18. Willerson JT, Ridker PM. Inflammation as a cardiovascular risk factor. Circulation 2004; 109: II2-10.

19. Pattanshetty DJ, Anna K, Gajulapalli RD, Sappati-Biyyani RR. Inflammatory bowel "Cardiac" disease: point prevalence of atrial fibrillation in inflammatory bowel disease population. Saudi J Gastroenterol 2015; 21: 325-9.

20. Frustaci A, Chimenti C, Bellocci F, Morgante E, Russo Ma, Maseri A. Histological substrate of atrial biopsies in patients with lone atrial fibrillation. Circulation 1997; 96: 1180-4.

21. Watanabe T, Takeishi Y, Hirono O, et al. C-reactive protein elevation predicts the occurrence of atrial structural remodeling in patients with paroxysmal atrial fibrillation. Heart Vessels 2005; 20: 45-9.

22. Gedikli O, Dogan A, Altuntas I, et al. Inflammatory markers according to types of atrial fibrillation. Int J Cardiol 2007; 120: 193-7.

23. Korantzopoulos P, Kolettis T, Siogas K, Goudevenos J. Atrial fibrillation and electrical remodeling: the potential role of inflammation and oxidative stress. Med Sci Monit 2003; 9: RA225-9.

24. Atreya R, Neurath MF. Involvement of IL-6 in the pathogenesis of inflammatory bowel disease and colon cancer. Clin Rev Allergy Immunol 2005; 28: 187-96.

25. Hirano T, Akira S, Taga T, Kishimoto T. Biological and clinical aspects of interleukin 6. Immunol Today 1990; 11: 443-9.

26. Ishihara K, Hirano T. IL-6 in autoimmune disease and chronic inflammatory proliferative disease. Cytokine Growth Factor Rev 2002; 13: 357-68.

27. Kishimoto T. Interleukin-6: from basic science to medicine: 40 years in immunology. Annu Rev Immunol 2005 23: $1-21$.

28. Mudter J, Neurath MF. II-6 signaling in inflammatory bowel disease: pathophysiological role and clinical relevance. Inflamm Bowel Dis 2007; 13: 1016-23.

29. Kristensen SL, Lindhardsen J, Ahlehoff O, et al. Increased risk of atrial fibrillation and stroke during active stag es of inflammatory bowel disease: a nationwide study. Europace 2014; 16: 477-84.

30. Harada M, Van Wagoner DR, Nattel S. Role of inflammation in atrial fibrillation pathophysiology and management. Circ J 2015; 79: 495-502.

31. Guo Y, Lip GY, Apostolakis S. Inflammation in atrial fibrillation. J Am Coll Cardiol 2012; 60: 2263-70.

32. Kourliouros A, Savelieva I, Kiotsekoglou A, Jahangiri M, Camm J. Current concepts in the pathogenesis of atrial fibrillation. Am Heart J 2009; 157: 243-52. 